\title{
Tuberculosis extrapulmonar
}

\section{Una perspectiva desde un hospital de tercer nivel}

\section{Extrapulmonary tuberculosis A perspective from a third-level hospital}

Manuel C. Pacheco Gallego, MD(1); Carlos Awad, MD(1); Gerson Arias, MD(2); Paulina Ojeda, MD(3); Manuel A. Garay, MD(1); Antonio Lara, MD(1); Liliana Arias, $\mathrm{MD}^{(4)}$

\section{RESUMEN}

Introducción: la tuberculosis sigue siendo una enfermedad de alta prevalencia y muestra, además, un aumento considerable en los pacientes VIH/SIDA; de igual manera, las formas extrapulmonares vienen tomando cada vez mayor relevancia. Se informan aproximadamente 9 millones de casos nuevos y más de 1 millón y medio de muertes cada año por tuberculosis, así mismo toma importancia cardinal el incremento en la incidencia de casos de tuberculosis extrapulmonar.

Objetivos: evaluar y presentar los datos de un hospital de tercer nivel en relación con tuberculosis extrapulmonar y ofrecer recomendaciones generales para su diagnóstico y tratamiento.

Diseño, materiales y métodos: se revisaron los registros clínicos y las bases epidemiológicas del programa de tuberculosis de los últimos 12 años de un hospital de tercer nivel de Bogotá, Colombia (Hospital Santa Clara ESE) y se hizo una revisión de la literatura en lo que respecta a la tuberculosis y su presentación extrapulmonar.

Resultados: en el consolidado de casos de tuberculosis extrapulmonar de la población del Hospital Santa Clara ESE de 12 años se obtuvieron $30 \%$ de casos extrapulmonares en pacientes no VIH/SIDA y $47 \%$ en pacientes con SIDA. Las formas de presentación extrapulmonar más frecuentes fueron la ganglionar, el compromiso del sistema nervioso central y la miliar.

Conclusiones: se encontró una importante incidencia en los casos extrapulmonares. El reto más importante en la tuberculosis extra-pulmonar radica en la sospecha clínica y en la selección del método para la confirmación diagnóstica. Esta formas son paucibacilares y de bajo contagio, pero pueden producir alta morbi-mortalidad, la misma que puede ser mayor en los pacientes con VIH/SIDA.

Palabras clave: tuberculosis, extrapulmonar, VIH/SIDA, Mycobacterium.

\section{ABSTRACT}

Introduction: the prevalence of tuberculosis (TB) continues to be high, and shows an important increase in patients with HIVIAIDS. The relevance of extrapulmonary forms of the disease has likewise become increasingly greater. Approximately 9 million new cases of tuberculosis are reported every year, with more than one and a half million deaths due to the disease during the same period of time.

Objectives: to evaluate and present the data of a third-level hospital with regard to extrapulmonary tuberculosis, and to give some general recommendations for its diagnosis and treatment.

Design, Materials and Methods: the clinical charts and the epidemiologic databases of the tuberculosis program carried

(1) Internista, Neumólogo.

(2) Internista, Infectólogo.

(3) Patóloga.

(4) Radióloga. Hospital Santa Clara. Bogotá, Colombia.

Correspondencia: Manuel Pacheco Gallego. Correo electrónico: mcpachecog@hotmail.com

Recibido: 12-05-2013. Aceptado: 23-06-2013. 
out over the past 12 years at a third-level hospital of Bogotá, Colombia (Hospital Santa Clara E.S.E.) were evaluated, and a review of the literature concerning tuberculosis and its extrapulmonary presentation was carried out.

Results: The consolidated data of cases of extrapulmonary tuberculosis among the population of the Hospital Santa Clara E.S.E. over a 12 -year period: $30 \%$ of extrapulmonary cases were found in non-HIVIAIDS-infection patients and $47 \%$ in patients with AIDS. The most common forms of extrapulmonary presentation were: of the lymph nodes, of the central nervous system, and of the miliary type.

Conclusions: An important incidence of extrapulmonary cases was found. The challenge with regard to extrapulmonary tuberculosis lies on clinical suspicion and on the selection of the method for diagnostic confirmation. These forms are paucibacillary and not very contagious, but can produce high morbidity and mortality rates, especially in patients with HIVIAIDS.

Keywords: tuberculosis, extrapulmonary, HIVIAIDS, Mycobacterium.

Rev Colomb Neumol 2013; 25 (1):16-26

\section{INTRODUCCIÓN}

El Mycobacterium tuberculosis es una bacteria ácido-alcohol resistente, aeróbica estricta. El crecimiento en colonias presenta un aspecto rugoso y de color blanco cremoso, con diámetros de dimensiones variables. Para su crecimiento depende de la presencia de oxígeno; es sensible al calor, la luz solar y la luz ultravioleta. Como característica importante tiene un periodo de multiplicación muy lento (divisiones celulares cada 16 a 20 horas) y capacidad de entrar en estado latente, circunstancia que tiene importante repercusión clínica en formas de presentación tardía y en fenómenos de reactivación cuando existen alteraciones en la inmunidad del huésped.

Para el diagnóstico microbiológico los medios de cultivo más usados son el de Lowenstein Jensen y el de Ogawa (figura 1). Una de las más grandes dificultades para el diagnóstico radica en que utilizando estos métodos convencionales se requieren como mínimo 15 días para la identificación del microorganismo y hasta ocho semanas de incubación.

El cuadro clínico del paciente con tuberculosis dependerá del órgano afectado y el estado de inmunidad; existen síntomas y signos que son generales y se encuentran en las diferentes formas de presentación de la enfermedad, como la pérdida progresiva de peso, la fiebre prolongada de origen desconocido y la diaforesis nocturna. En el caso de la tuberculosis extrapulmonar su mayor dificultad radica en que esta presentación es paucibacilar, lo que limita aún más la posibilidad de obtener resultados en estudios básicos como baciloscopias y por lo general se requiere esperar reportes de cultivos o de patología. Ya que la concentración más baja de microorganismos que se
¿Qué se conocía previamente del tema?

- La tuberculosis extrapulmonar supone 10 a $20 \%$ del total de los casos de tuberculosis en inmunocompetentes, y se incrementa notablemente en inmunodeficiencia. En SIDA hasta $60 \%$ de los casos pueden estar asociados a tuberculosis extrapulmonar.

¿Qué aporta el estudio?

- En los últimos 12 años, en un programa de tuberculosis de un hospital de referencia en Bogotá, se registraron $30 \%$ de casos de tuberculosis extrapulmonar en pacientes no VIH/SIDA y $47 \%$ en pacientes con SIDA. Las formas de presentación extrapulmonar más frecuentes fueron la ganglionar, el compromiso del sistema nervioso central y la miliar.

puede detectar en la baciloscopia es de $10.000 / \mathrm{mL}$ de muestra, en realidad, sólo puede detectar tuberculosis avanzadas o multibacilares (con alta carga bacilífera).

Puede decirse que los síntomas carecen de alta especificidad y pueden ser similares a los de otras patologías infecciosas. En general el curso clínico de la enfermedad es insidioso y puede tardar varios meses en manifestarse con síntomas claros, de ahí que se requiera una alta sospecha diagnóstica y la identificación de estudios adecuados para su confirmación.

Los síntomas que se evidencian son los que se encuentran en relación con una enfermedad crónica debilitante dados por fiebre y diaforesis nocturna, pérdida de peso, anorexia, astenia y adinamia. La primoinfección también puede ser inespecífica.

Existen formas de presentación más agresivas dadas por hemoptisis con cuadros similares a neumonía donde puede haber diseminación pulmonar 


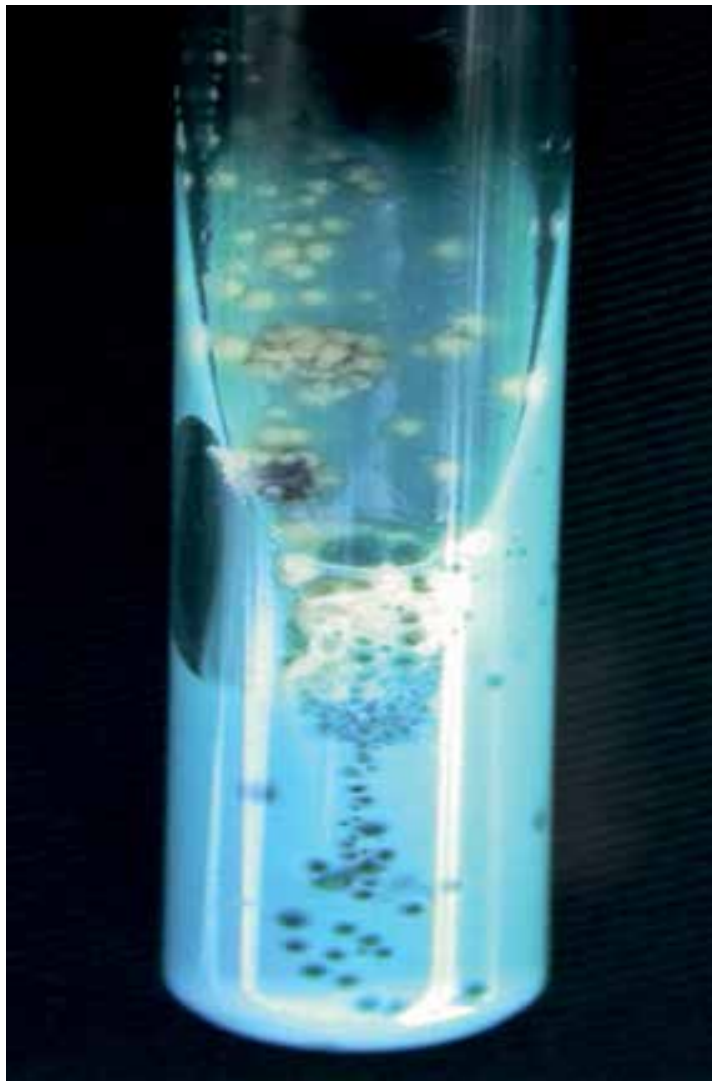

Figura 1. Crecimiento de colonias en cultivo de Ogawa.

Fuente: Laboratorio de tuberculosis. Hospital Santa Clara.

extensa o incluso diseminación hematógena con relación a la denominada tuberculosis miliar.

La mayoría de las revisiones y estudios clínicos consideran el pulmón como el sitio de ingreso para la diseminación de la enfermedad y la generación del compromiso extrapulmonar; las vías principales identificadas son la hematógena, linfática y por contigüidad (1). Prácticamente la totalidad de la economía corporal puede ser comprometida dejando la tuberculosis como una enfermedad sistémica, más que una patología de localización exclusiva pulmonar. Las localizaciones más frecuentes incluyen: sistema nervioso central, ganglionar, pleural, genitourinaria, piel, ósea, laríngea, pericárdica y gastrointestinal.

\section{EPIDEMIOLOGÍA}

La tuberculosis es una de las enfermedades que acompaña al ser humano desde épocas remotas, tal como se ha confirmado con el hallazgo de lesiones tuberculosas en momias egipcias y precolombinas.
El punto más alto en la epidemia de tuberculosis se alcanzó a finales del siglo XVIII en Inglaterra, a principios del siglo XIX en Europa occidental y a finales del siglo XIX en Europa Oriental y América del Norte y del Sur (2), pero todavía es una enfermedad infectocontagiosa de prevalencia alta e incidencia alarmante en regiones como África y Asia.

Se informan aproximadamente 9 millones de casos nuevos y más de un millón y medio de muertes cada año por tuberculosis (3). Estadísticas revelan una importante patología de presentación global y de alto impacto en la salud pública. Por ello los planes de salud de la mayoría de países tienen estrategias para reducir la prevalencia de tuberculosis; los planteamientos propuestos para las metas de salud mundial y desarrollo del milenio incluyen disminución del $50 \%$ de las muertes por tuberculosis en el 2015 (4).

La tuberculosis es una patología infecciosa de origen bacteriano y de carácter contagioso. Es causada por el Mycobacterium tuberculosis o bacilo de Koch, perteneciente al complejo Mycobacterium. Como ya se había mencionado, es una enfermedad que puede tener compromiso sistémico o localizado.

Es importante reconocer el impacto del VIH en la incidencia de casos de tuberculosis. En este sentido, se estimaron 4.9 millones de casos nuevos de tuberculosis en pacientes con VIH para 2004, donde las tasas más altas de coinfección se reportaron en África y el Sureste asiático. En América se aprecia una incidencia aproximada del $4 \%$, que varía entre las diversas regiones (5).

\section{EPIDEMIOLOGÍA EN AMÉRICA Y COLOMBIA}

La región de las Américas notificó en 2003, 227.551 casos de tuberculosis de todos los tipos, con una tasa de 26/100.000 habitantes. Para ese mismo año, Brasil notificó 83.575 casos nuevos, correspondientes a una incidencia de 47,3/ 100.000 habitantes, posicionándose como uno de los principales países en Suramérica (6).

Colombia reporta anualmente más de 11.000 casos nuevos; durante el año 2008 se notificaron 11.342 casos nuevos, para una incidencia de 25,6 casos por 100.000 habitantes, de los cuales 6.815 $(60,08 \%)$ ocurrieron en hombres y 4.527 en mujeres $(39,91 \%)$. En relación a la tuberculosis infantil se informaron 719 casos $(6,3 \%)$ en población menor de 15 años, para una incidencia de 5,47 casos por 100.000 menores de 15 años (3). 
Analizando los casos notificados entre 1993 y 2008, se hizo una agrupación de las entidades territoriales como zonas de riesgo muy alto (por encima de 41,39 casos por 100.000 habitantes), o alto riesgo (entre 30,15 y 41,39 casos por 100.000 habitantes). Las regiones más afectadas son: Orinoquía, Amazonía, Chocó, Quindío y La Guajira.

En cuanto al VIH, existen datos de 5.537 casos anuales para un $6,8 \%$ de coinfección, donde $15,6 \%$ están en relación con tuberculosis extrapulmonar (7).

\section{EPIDEMIOLOGÍA DE LA TUBERCULOSIS EXTRAPULMONAR}

Supone el 10\%-20\% del total de los casos de tuberculosis en inmunocompetentes, y se incrementa notablemente en inmunodeficiencia. En SIDA hasta en $60 \%$ de casos pueden estar asociados a tuberculosis extrapulmonar. Las tuberculosis extrapulmonares tienen en general baciloscopia negativa, con baja capacidad de contagio y difícil diagnóstico (8).

El compromiso por tuberculosis extrapulmonar muestra un crecimiento durante las últimas décadas, partiendo de un estimado en Asia de $1,2 \%$ en la década de los 60 y de $8 \%$ en América para la misma época, hasta llegar a cifras de $6,7 \%$ y $17 \%$ respectivamente para los 90 (9).

La tuberculosis pleural se describe como una de las formas de localización más frecuentes en el mundo y ésta le sigue la localización linfática; ambas constituyen la formas de presentación más frecuentes en pacientes jóvenes (10). El resto de posibles localizaciones de la tuberculosis extrapulmonar es menos común, aunque pueden presentarse y con mayor incidencia en pacientes portadores de $\mathrm{VIH}$.

En Bogotá, el estudio de Fuentes y Villamil en el que se describió tuberculosis pulmonar y extrapulmonar, reportó un total de 3.687 casos entre 2005 y 2008 , 1.382 de los cuales fueron formas extrapulmonares, lo cual corresponde al $37,5 \%$ y confirma una forma de presentación importante que obligaría al médico a incluirla dentro de los diagnósticos diferenciales cuando se enfrente a casos sospechosos (11).

En este grupo de pacientes la forma extrapulmonar tuvo mayor prevalencia en hombres entre 26 a 50 años. Las presentaciones comúnmente identificadas correspondieron a la pleural, en $14 \%$, a la meníngea, en $11,4 \%$ (un nivel superior a lo reportado en otros estudios) y a la miliar, en $7,5 \%$. Estos datos permiten contar con un estimado para orientar la forma de enfrentar esta patología.

\section{HALLAZGOS EN EL HOSPITAL SANTA CLARA}

El seguimiento en el programa de tuberculosis del Hospital realiza una vigilancia a los casos pulmonares y extrapulmonares; a continuación se exponen los principales hallazgos en relación con el tópico que está en discusión.

En el período de doce años evaluado se presentaron en total 1.774 casos, de los cuales 227 correspondía a pacientes con VIH y de éstos la mayoría al género masculino (tabla 1).

En relación con la ubicación, tanto en los pacientes generales como en aquellos con SIDA la primera ubicación corresponde a la pulmonar en $70,9 \%$ y $52,9 \%$ respectivamente, pero es importante evidenciar como en el SIDA las presentaciones extrapulmonares ocupan aproximadamente un $47 \%$ frente a los pacientes no SIDA donde tiene un porcentaje menor (tabla 2).

Dentro de las formas extrapulmonares las más frecuentes identificadas en pacientes sin SIDA son: pleural, miliar, ganglionar y del sistema nervioso central. En el grupo de pacientes con SIDA hay una variación y fue más frecuente la presentación miliar, seguida por la ganglionar y la del sistema nervioso central. Dentro de la presentación en las clasificadas como otras se incluyen ósea e intestinal que también

Tabla 1. Programa de tuberculosis - Hospital Santa Clara.

\begin{tabular}{|l|c|c|c|c|c|}
\hline \multirow{2}{*}{ Sexo } & \multicolumn{2}{|c|}{$\begin{array}{c}\text { Total casos } \\
\text { tuberculosis }\end{array}$} & \multicolumn{2}{c|}{$\begin{array}{c}\text { Total casos } \\
\text { tuberculosis - SIDA }\end{array}$} & $\begin{array}{c}\text { Porcentaje casos } \\
\text { SIDA/ } \\
\text { tuberculosis }\end{array}$ \\
\cline { 2 - 5 } & No. & $\%$ & No. & $\%$ & 16,8 \\
Masculino & 1.145 & 64,5 & 192 & 84,6 & 5,6 \\
Femenino & 629 & 35,5 & 35 & 15,4 & \\
Total & 1.774 & 100 & 227 & 100 & \\
\hline
\end{tabular}


Tabla 2. Programa de tuberculosis - Hospital Santa Clara.

\begin{tabular}{|l|r|r|r|r|c|}
\hline \multirow{2}{*}{ Localización } & \multicolumn{2}{|c|}{$\begin{array}{c}\text { Total casos } \\
\text { tuberculosis }\end{array}$} & \multicolumn{2}{c|}{$\begin{array}{c}\text { Total casos } \\
\text { tuberculosis - SIDA }\end{array}$} & $\begin{array}{c}\text { Porcentaje casos } \\
\text { SIDA/ } \\
\text { tuberculosis }\end{array}$ \\
\cline { 2 - 5 } & No. & $\%$ & No. & $\%$ & 9,5 \\
Pulmonar & 1.258 & 70,9 & 120 & 52,9 & 31,2 \\
Miliar & 109 & 6,1 & 34 & 15,0 & 6,3 \\
Pleural & 191 & 10,8 & 12 & 5,3 & 36,2 \\
Ganglionar & 69 & 3,9 & 25 & 11,0 & 35,3 \\
Meningocerebral & 68 & 3,8 & 24 & 10,6 & 15,2 \\
Otras & 79 & 4,5 & 12 & 5,3 & 12,8 \\
TOTAL & 1.774 & 100,0 & 227 & 100 & \\
\hline
\end{tabular}

se han identificado en el seguimiento del grupo de tuberculosis del hospital (figuras 2 a 5).

En lo concerniente al éxito de la terapia, debido a las características sociodemográficas de los pacientes incluidos, las tasas de abandono fueron del $14,4 \%$, y se hallaron cifras considerables de mortalidad del $16 \%$ en casos sin SIDA. Sin embargo, llama la atención cómo en pacientes con SIDA la mortalidad puede llegar hasta el $42,3 \%$, situación que hace imprescindible incluir dentro de las posibilidades diagnósticas de estos pacientes la existencia de tuberculosis y recordar que las formas extrapulmonares en SIDA en este análisis, alcanzaron en cifras estadísticas de hasta el $47 \%$.

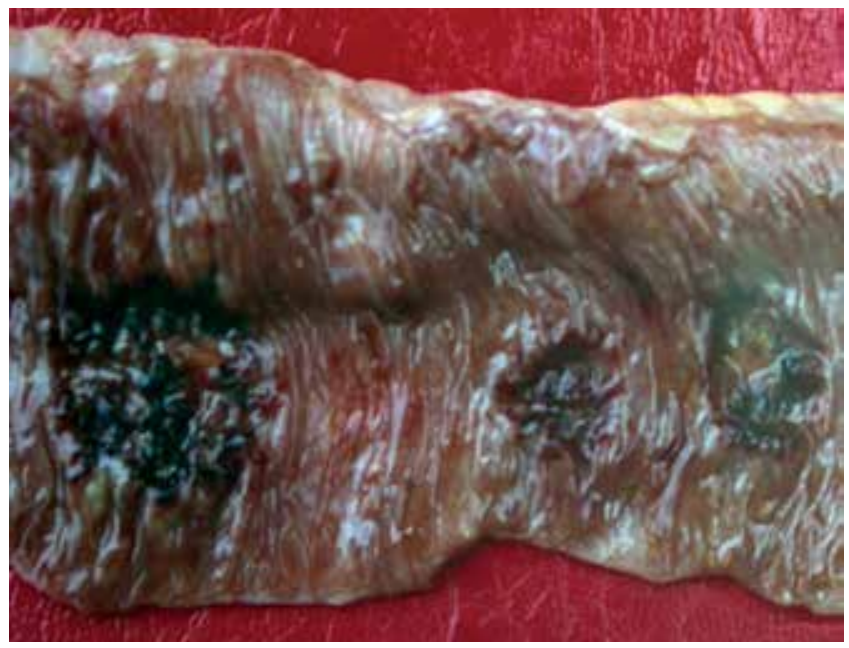

Figura 2. Segmento de intestino delgado que muestra úlceras de fondo hemorrágico por tuberculosis.

Fuente: Hospital Santa Clara, Departamento de Patología.
Con base en lo descrito se hace una revisión de las generalidades de las tres formas más frecuentemente encontradas en el Hospital Santa Clara y luego se dan recomendaciones precisas sobre el tratamiento y la utilidad de nuevos métodos diagnósticos.

\section{TUBERCULOSIS GANGLIONAR}

M. tuberculosis tiene la capacidad de diseminarse por vía linfática, lo que favorece esta presentación; es considerada, además, la forma por la cual se da el compromiso ganglionar. Yew sugiere que la vía predominante de propagación del bacilo proviene del drenaje del parénquima pulmonar por los linfáticos del

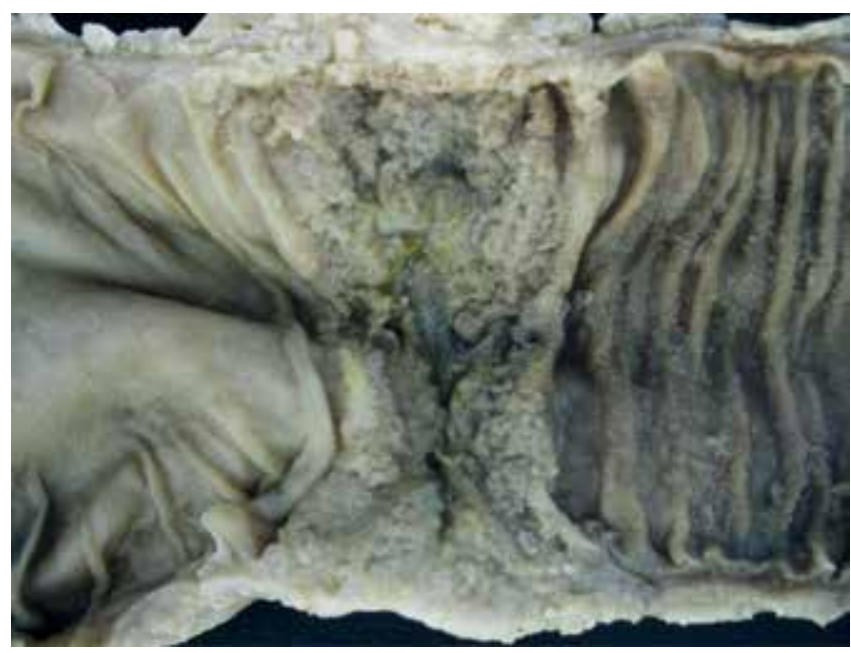

Figura 3. Segmento de colon que muestra úlcera de fondo necrótico con retracción de la pared por tuberculosis.

Fuente: Hospital Santa Clara, Departamento de Patología. 


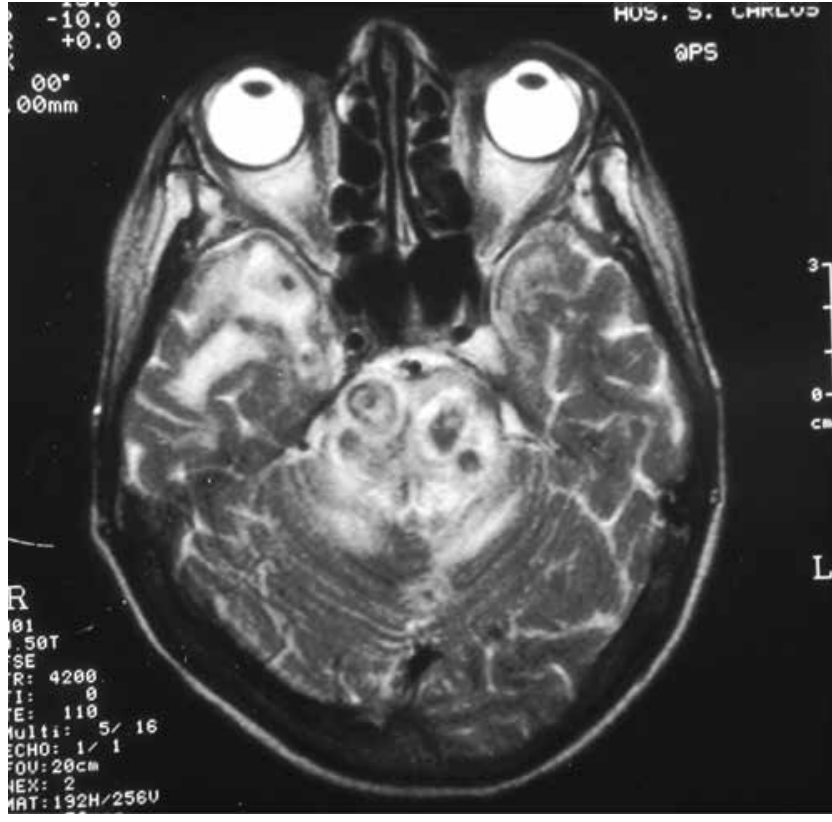

Figura 4. Secuencias axiales sopesadas en T2. Múltiples lesiones supra e infratentoriales con centro hipointenso y halo hiperintenso. Efecto de masa y edema perilesional.

Fuente: Hospital Santa Clara, Departamento de Patología.

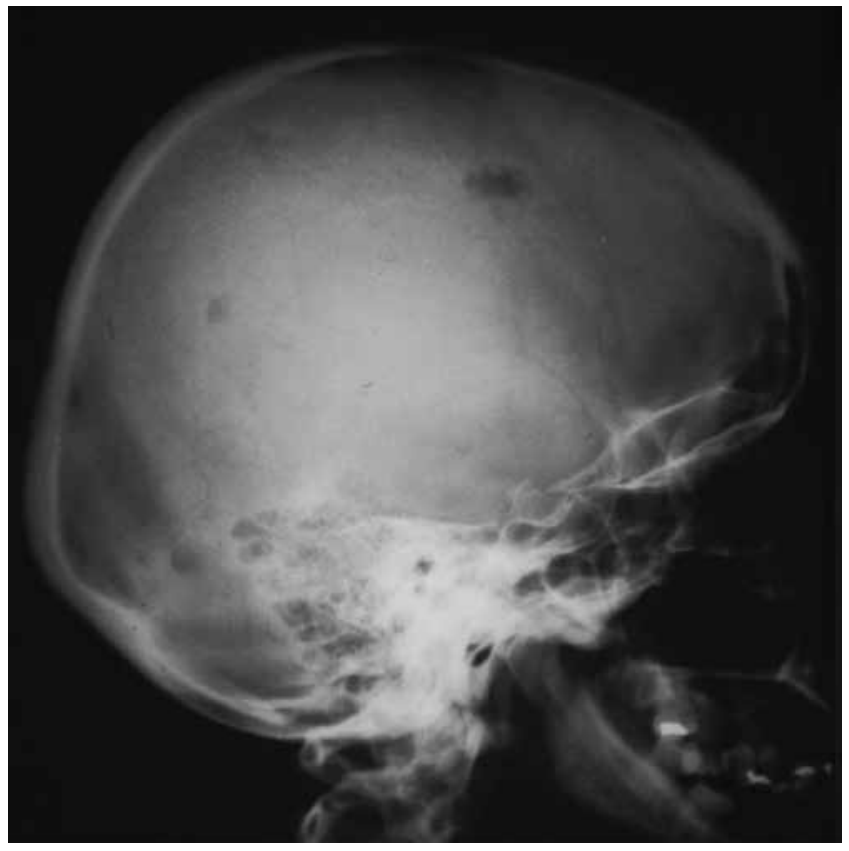

Figura 5. Lesiones líticas en cráneo. En un paciente con tuberculosis ósea.

Fuente: Hospital Santa Clara, Departamento de Patología. pulmón derecho y el lóbulo inferior del pulmón izquierdo que van hacia a los ganglios linfáticos supraclaviculares derechos y luego a la cadena cervical. Los hallazgos en las radiografías de tórax mostraron que el $41,4 \%$ de los pacientes con linfadenitis tuberculosa tenían evidencia de tuberculosis pulmonar, y el $22,5 \%$ de ellos tenían enfermedad activa con base en las pruebas radiográficas (12).

Como lo menciona Handa en su revisión, en esta presentación ocurrió un incremento desde la década de los 80 probablemente en relación con el aumento en los casos de SIDA, siendo más común en grupos de pacientes jóvenes de sexo masculino (hallazgo que también se ha comprobado en el Hospital Santa Clara), en contraposición con otras regiones del mundo, como India, donde se ha visto que las mujeres son las más afectadas (13).

El compromiso puede darse en cadenas ganglionares periféricas (escrófula), y otro grupo que afecta principalmente cadenas ganglionares internas. El compromiso periférico compromete básicamente adenopatías de cabeza y cuello (figura 6), por lo que debe incluirse la tuberculosis dentro de la evaluación diagnóstica de estos pacientes. Son menos frecuentes los compromisos de la cadena ganglionar inguinal, si bien en pacientes con SIDA estas localizaciones pueden variar.

Usualmente se observan adenopatías de crecimiento lento, en algunos casos asintomáticas y en algún momento de la evolución se pueden observar síntomas sistémicos con fiebre, pérdida de peso, fatiga y diaforesis nocturna ocasional. Los pacientes VIH positivos suelen ser más sintomáticos. Como se mencionó, es importante recordar que viene dándose un aumento de los casos producidos por micobacterias no tuberculosas tanto en la población general como en los pacientes con SIDA, siendo frecuente el compromiso por $M$. avium y en menor proporción por M. scrofulaceum (14), donde el triángulo yugular posterior o los ganglios linfáticos supraclaviculares resultan ser los sitios más afectados (15).

El compromiso ganglionar interno puede llegar a producir grandes masas en el mediastino, que en algunos casos pueden comprimir e incluso perforar el árbol traqueobronquial. Está cada vez más rara, guarda relación con la mayor disponibilidad de tratamiento efectivo. El siguiente grupo de localización corresponde a las cadenas ganglionares abdominales, donde la presentación es variada y no existe 


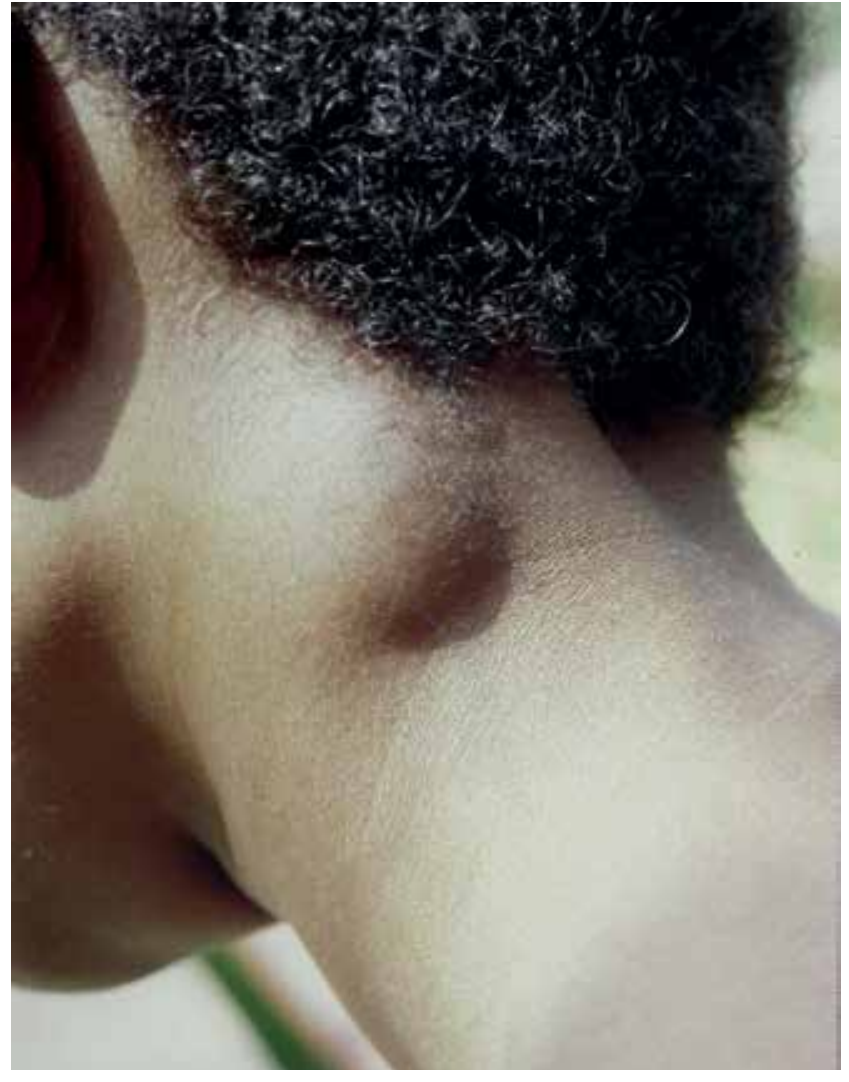

Figura 6. Adenopatía cervical en un caso de tuberculosis ganglionar.

Fuente: Departamento de Patología, Hospital Santa Clara.

uniformidad en relación con el tamaño de las lesiones ni con las características patognomónicas de la enfermedad (figura 7).

Para el diagnóstico adecuado, además de una importante sospecha clínica, es fundamental el estudio anatomopatológico. Los métodos diagnósticos recomendados incluyen citología por aspiración con aguja fina, biopsia por tru-cut y biopsia excisional. Teniendo en cuenta que se requieren muestras para estudio patológico y cultivo, se recomienda tomar la mejor cantidad de tejido con el objetivo de favorecer la posibilidad de un diagnóstico acertado.

\section{TUBERCULOSIS DE SISTEMA NERVIOSO CENTRAL}

Las formas de aparición son variadas e incluyen dos grupos: compromiso intracraneal que a su vez incluye:

- Meningitis tuberculosa.

- Encefalitis tuberculosa.

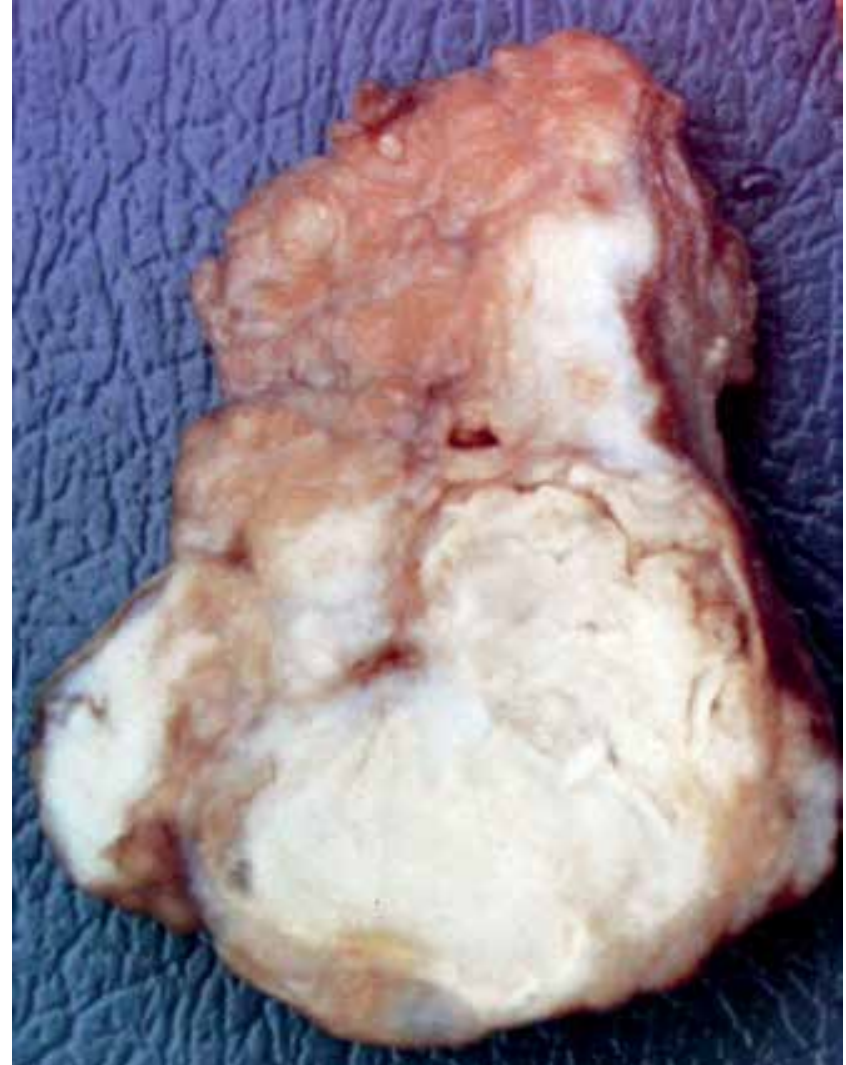

Figura 7. Ganglio mesentérico, aumentado de tamaño con extensa necrosis caseosa al corte.

- Vasculopatía tuberculosa.

- Tuberculoma.

- Absceso tuberculoso.

Así mismo, existe un grupo de pacientes con compromiso extracraneal donde puede desarrollarse tuberculoma no óseo y meningitis espinal, y en algunos casos paraplejía con o sin asociación a mal de Pott (16).

Los principales factores de riesgo para desarrollar tuberculosis del sistema nervioso central son la coinfección con $\mathrm{VIH}$, la malnutrición, la historia de neoplasias y el uso de agentes inmunosupresores (17).

Desde el punto de vista clínico se ha clasificado en tres grupos (18):

1. Alerta y orientado sin déficit focal.

2. Pacientes con escala de Glasgow de 11 a 14, ó 15 si hay déficit neurológico focal. 
3. Escala de Glasgow menor o igual a 10 con o sin déficit neurológico focal.

Los síntomas más descritos incluyen cefalea, vómito, alteración del sensorio $\mathrm{y}$, en ocasiones, déficit neurológico focal. El estudio del líquido cefalorraquídeo es fundamental para el diagnóstico; en general presenta pleocitosis con predominio linfocitario, de mayor sensibilidad cuando supera el $60 \%$. También se encuentra hiperproteinorraquia con cifras superiores a $100 \mathrm{mg} / \mathrm{dL}$, con disminución de la glicemia en el LCR y estudios negativos para otras patologías que pueden comprometer el sistema nervioso central, incluida la citología para neoplasias (19). La duración de los síntomas también es importante; en general, las agudas de menos de seis días no son frecuentes y la mayoría corresponde a casos sub-agudos.

Los hallazgos imaginológicos más comunes incluyen: realce meníngeo basal, hidrocefalia, tuberculoma e hiperdensidad basal pre-contraste.

Se han diseñado diversas escalas de clasificación para orientar al diagnóstico definiéndola como: definitiva, en relación con la confirmación microbiológica que puede ser por baciloscopia, PCR, cultivos o hallazgos anatomopatológicos, probable, posible y meningitis no tuberculosa dependiendo de los hallazgos obtenidos en la evaluación clínica, el LCR, las imágenes del sistema nervioso central, la presencia de tuberculosis pulmonar, cambios radiográficos del tórax sugestivos de tuberculosis y la exclusión de otros diagnósticos.

Es importante recordar que la parálisis de pares craneales se produce en el $20 \%$ al $30 \%$ de los casos y puede ser la manifestación inicial; el sexto par craneal es más afectado. La pérdida de visión debido a la afectación del nervio óptico puede ocasionalmente ser una condición dominante (20).

La utilidad de métodos diagnósticos alternativos en el estudio del LCR incluye básicamente dos elementos en la mayoría de las revisiones, PCR y ADA. Un meta-análisis publicado en Lancet en relación con el uso de PCR, método de amplificación de ácidos nucleicos (NAA) para el diagnóstico de tuberculosis meningea (TBM) mostró baja sensibilidad, de aproximadamente $56 \%$, pero especificidad de $98 \%$, si se tiene en cuenta que la rentabilidad aumenta con grandes volúmenes de LCR (21).
En relación con ADA, esta es una enzima del metabolismo de purinas, que desamina adenosina para la conversión a inosina. La presencia de ADA está asociada a proliferación linfocítica y se considera como un marcador de inmunidad celular.

Se han realizado varios estudios para evaluar la utilidad de ADA en el líquido cefalorraquídeo (CSF) para mejorar diagnóstico de TBM. Las sensibilidades y especificidades varían desde $44 \%$ hasta $100 \%$ y de $71 \%$ a $100 \%$, respectivamente. Algunos trabajos mostraron diferenciación estadísticamente significativa para distinguirla de la meningitis bacteriana. Puede ser una estrategia útil sobre todo con puntos de corte elevados (22). Es importante recordar que el punto de corte a tener en cuenta actualmente es de $9 \mathrm{UI} / \mathrm{L}$ y que en pacientes con VIH esta aproximación puede variar debido a la frecuente coinfección con otros gérmenes que pueden afectar la prueba.

\section{TUBERCULOSIS MILIAR}

Tuberculosis miliar es reconocida como una forma potencialmente letal de la tuberculosis en relación con la diseminación masiva del bacilo. Se evidencia además un incremento en su presentación en asociación con el aumento de los casos de SIDA y el aumento en la utilización de fármacos inmunosupresores.

En esta forma de la enfermedad las presentaciones atípicas son frecuentes y el diagnóstico diferencial es amplio. Es frecuente la presentación grave con casos que llegan hasta el síndrome de dificultad respiratoria aguda. El aislamiento de $M$. tuberculosis a partir de fluidos corporales, esputo, o los especímenes de biopsias, examen aplicación de métodos moleculares tales como PCR, son requeridos para la confirmación del diagnóstico (23).

Los factores de riesgo asociados incluyen SIDA, malnutrición, alcoholismo, diabetes, falla renal crónica, pacientes trasplantados, portadores de enfermedad del colágeno, presencia de silicosis y uso de medicamentos inmunosupresores (24).

La presentación clínica es variada e incluye síntomas generales y específicos en relación con los órganos más comprometidos; de manera universal, se considera tuberculosis diseminada cuando se comprometen más de dos órganos. Lo más frecuente es la presencia de fiebre de varias semanas 
de duración, anorexia, pérdida de peso, astenia, adinamia y tos predominantemente seca. Especialmente entre las personas mayores y las severamente inmunodeprimidas se presenta emaciación progresiva.

Con la disponibilidad de la tomografía computarizada de alta resolución el diagnóstico se puede orientar con mayor facilidad; en ésta se evidencia un patrón micronodular difuso.

En un estudio previo del Hospital Santa Clara en el que se evaluaron las necropsias de pacientes con SIDA y tuberculosis, la asociación con tuberculosis miliar mostró que el compromiso principal era hepático y comprometía al $100 \%$ de los pacientes, al igual que al bazo, seguido por el compromiso pulmonar en el $80 \%$ de los casos y el de la médula ósea en el $70 \%$; los órganos menos comprometidos fueron el sistema nervioso central y la pleura con $30 \%$.

\section{UTILIDAD DEL XPERT MTB/RIF EN TUBERCULOSIS EXTRAPULMONAR}

Como se ha descrito, la oportunidad de obtener un diagnóstico rápido de la tuberculosis es fundamental con miras a lograr curar la enfermedad en el paciente y limitar su propagación. En relación a las formas extrapulmonares, si bien son de bajo contagio tienden a ser más agresivas o a generar mayores secuelas que pueden limitar de manera significativa la calidad de vida. Los métodos actuales de diagnóstico para la tuberculosis extrapulmonar requieren, en su gran mayoría, cultivos que pueden tardar entre 6 a 8 semanas para una adecuada identificación, ya que como se indicó antes estas presentaciones son paucibacilares. Debido a lo anterior se presenta evidencia con técnicas rápidas de diagnóstico, tal vez dentro de las más accesibles en Colombia en un futuro no muy lejano el Xpert MTB/RIF. Así pues se expondrán algunas consideraciones al respecto.

Esta técnica consiste en una prueba automatizada molecular para Mycobacterium tuberculosis que tiene la capacidad de identificar la resistencia a la rifampicina (R). Utiliza PCR en tiempo real para amplificar una secuencia del gen rpoB. Ha mostrado sensibilidad y especificidad del $95 \%$ en el diagnóstico de formas pulmonares (25).

Fue aprobada por la Organización Mundial de la Salud (OMS) para el diagnóstico de tuberculosis en países de baja y media prevalencia (26).
En el estudio evaluado por Russo y Piersimoni se revisó su utilidad en muestras diferentes a esputo en un publicación de 2013 , donde se probó la técnica con líquido pleural, pus, biopsias de tejido obtenidas por BACAF, aspirado gástrico, orina y líquido pericárdico y peritoneal, comparados contra cultivos.

Se evaluaron 268 muestras de tuberculosis extrapulmonar en una gama de sitios anatómicos identificando una sensibilidad de $81,3 \%$; $95 \%$ : IC 76,2-85,8 y los datos de 1.206 muestras en las que se excluyó la tuberculosis extrapulmonar (especificidad: 99,8\%, 95\% IC: 99,4 a 100) (10).

De igual manera en otro estudio de Clemente y Palacios llevado a cabo en España, se evaluaron muestras extrapulmonares en las que se halló sensibilidad global Xpert MTB/RIF para el diagnóstico de tuberculosis de un 73,6\%, especificidad del $99,9 \%$, VPP $96,3 \%$ y VPN de $98,7 \%$. La prueba identifica correctamente el $98,7 \%$ de las muestras extrapulmonares (27).

Lo descrito evidencia el papel preponderante que desempeñará esta prueba en el diagnóstico rápido no sólo de tuberculosis pulmonar sino extrapulmonar, con muestras de diferentes tejidos.

\section{TRATAMIENTO Y RECOMENDACIONES GENERALES}

Básicamente puede mencionarse que el tratamiento de las formas extrapulmonares sigue los mismos principios que los de la tuberculosis pulmonar; las diferencias principales radican en la duración y la utilidad de los esteroides.

En la mayoría de las localizaciones de tuberculosis extrapulmonar son formas con escaso número de bacilos, que dificultan la selección de los mutantes resistentes naturales, de tal manera que pareciera no necesario prolongar el tratamiento; sin embargo, persiste la controversia en las formas meníngeas, osteoarticular y ganglionar (28).

Acerca de la penetración de los fármacos antituberculosos al sistema nervioso central es claro que es del $100 \%$ para isoniazida $(H)$, pirazinamida $(Z)$ y etionamida $(E)$, siendo menor para rifampicina $(R)$ que se estima en $57 \%$. De igual forma, teniendo en cuenta la cantidad de este medicamento que viaja no unido a proteínas, su penetración es útil en LCR (29). 
La publicación con las recomendaciones del Centro para Control y Prevención de Enfermedades (CDC), la Asociación Americana del Tórax (ATS) y la Asociación Americana de Enfermedades Infecciosas (IDSA) recomienda extender la terapia de 6 a 9 meses en las formas de compromiso osteoarticular y de 9 a 12 meses en el compromiso del sistema nervioso central, a fin de disminuir la posibilidad de recaídas, aunque por lo comentado previamente se esperaría que no fueran frecuentes. Con relación al uso de esteroides, la evidencia reportada recomienda su uso solo en formas de tuberculosis pericárdica y del sistema nervioso central (30).

Es importante recordar que hay un aumento de los casos producidos por micobacterias no tuberculosas tanto en la población general como en los pacientes con SIDA, siendo frecuente el compromiso por M. avium y, en menor proporción, por M. scrofulaceum (14).

\section{CONCLUSIONES}

El reto en la tuberculosis extra-pulmonar radica en la sospecha clínica y en la selección del método para la confirmación diagnóstica. Esta formas son paucibacilares y de bajo contagio pero pueden producir alta morbi-mortalidad, más aun en pacientes con VIH/SIDA, en quienes su presentación es más frecuente y agresiva. En general, requiere mayores estudios invasivos ya que las baciloscopias no son diagnósticas en la mayoría de veces. Además, teniendo en cuenta que se requiere un diagnóstico ágil, las nuevas técnicas rápidas empiezan a ganar protagonismo en este diagnóstico. Las bases del tratamiento son similares a las de la forma pulmonar, con variaciones en su duración y en el uso de esteroides asociados, que se recomiendan en la forma pericárdica y en el compromiso del sistema nervioso central.

\section{CONFLICTO DE INTERESES}

Los autores del estudio declaran no tener conflictos de interés.

\section{BIBLIOGRAFÍA}

1. Elder NC. Extrapulmonary tuberculosis. A review. Arch Fam Med. 1992; 1: 91-98.

2. Carvalho F. de M. Predicción de la tuberculosis. BMC Public Health. 2006; 6: 43-43.
3. Plan estratégico Colombia libre de tuberculosis 20102015. Ministerio de Protección Social. Disponible en: http:// www.paho.org/col/index.php?option=com_content\&view $=$ article \&id=431: plan-estrategico-colombia-libre-de-tuberculosis-2010-2015 \& catid=686\&ltemid=361.

4. Ayles $\mathrm{KH}$. How can we reduce TB deaths? Int J Tuberc Lung Dis. 2007; 11: 827.

5. Sharma SK, Mohan A, Kadhiravan T. HIV-TB co-infection: Epidemiology, diagnosis and management. Indian J Med Res. 2005; 121: 550-67.

6. Vendramini S, Villa T, Santos M, Gazetta C. Current epidemiological aspects of tuberculosis and the impact of the DOTS strategy in disease control. Rev Latino-Am Enfermagem. 2007; 15: 171-173.

7. Castiblanco CA, Ribón W. Coinfección de tuberculosis en pacientes con VIH/SIDA: un análisis según las fuentes de información en Colombia. Infectio. 2006; 10: 232-242.

8. Caminero JA, Rodríguez de Castro F, González A, Fernández JM, Juliá G, Cabrera P. Tuberculosis. 2011; 26: 23-27.

9. Global burden of tuberculosis: estimated incidence, prevalence and mortality by country. World Health Organization Global Surveillance and Monitoring Project. J Am Med Assoc. 1999; 282: $677-86$

10. Caminero JA, Rodríguez de Castro F, González A, Fernández JM, Juliá G, Cabrera P. Tuberculosis. 2011; 26: 45-66.

11. Fuentes J, Villamil N. Factores asociados a tuberculosis extrapulmonar en Bogotá D.C.. Trabajo de Tesis para optar al título de Especialista en Epidemiología. Bogotá: Universidad Colegio Mayor de Nuestra Señora del Rosario; 2005-2008.

12. Yew WW, Lee J. Pathogenesis of cervical tuberculous lymphadenitis: pathways to apical localization. Tuber Lung Dis. 1995; 76: $275-276$.

13. Handa U, Mundi I, Mohan S. Review article on tuberculous lymphadenitis. J Infect Dev Ctries. 2012; 6: 6-12.

14. Caminero J A, Rodríguez de Castro F, González A, Fernández JM, Juliá G, Cabrera P. Tuberculosis. 2011; 26: 28-44.

15. Kumar A. Lymph node tuberculosis. In: Sharma SK, Mohan A. (eds). Tuberculosis. 2nd. ed. New Delhi: Jaypee Brothers Medical Publishers; 2009. p. 397-409.

16. Cherian A, Thomas SV. Central nervous system tuberculosis. Afr Health Sci. 2011; 11: 116-27.

17. Phypers M, Harris T, Power C. CNS tuberculosis: a longitudinal analysis of epidemiological and clinical features. Int $\mathrm{J}$ Tuberc Lung Dis. 2006; 10: 99-103.

18. Thwaites GE, Tran TH. Tuberculous meningitis: many questions, too few answers. Lancet Neurol. 2005; 4: 160-70.

19. Ahuja GK, Mohan KK, Prasad K, Behari M. Diagnostic criteria for tuberculous meningitis and their validation. Tubercle Lung Dis. 1994; 75: 149-52.

20. Berger JR. Tuberculous meningitis. Curr Opin Neurol. 1994; 7: 191-200.

21. Pai M, Flores LL, Pai N, Hubbard A, Riley LW, Colford JM. Diagnostic accuracy of nucleic acid amplification tests for tu- 
berculous meningitis: a systematic review and meta-analysis. Lancet Infect Dis. 2003; 3: 633-43.

22. Rock RB, Olin M, Baker CA, Molitor TW, Peterson PK. Central nervous system tuberculosis: pathogenesis and clinical aspects. Clin Microbiol Rev. 2008; 21: 243-61.

23. Sharma SK, Mohan A, Sharma A, Mitra DK, Alladi M. Miliary tuberculosis: new insights into an old disease. Lancet Infect Dis. 2005; 5: 415-30.

24. Hussain SF, Irfan M, Abbasi M, et al. Clinical characteristics of 110 miliary tuberculosis patients from a low HIV prevalence country. Int J Tuberc Lung Dis. 2004; 8: 493-99.

25. Theron G, Peter J, van Zyl-Smit R, Mishra H, Streicher E, Murray S, Dawson R, et al. Xpert MTB/RIF versus sputum smear microscopy: microscopy needs a level playing Field. Am J Respir Crit Care Med. 2011; 184: 132-140.
26. Lawn, SD. Zumla, Al. Diagnosis of extrapulmonary tuberculosis using the $\mathrm{Xpert}^{\circledR}$ MTB/RIF assay. Expert Rev. Anti Infect Ther. 2012; 10: 631-635.

27. Clemente MG. Accuracy of the Xpert MTB/RIF test for rapid diagnosis of extrapulmonary tuberculosis. Am J Respir Crit Care Med. 2012; 185: A4717.

28. Caminero J A, Rodríguez de Castro F, González A, Fernández J M, Juliá G, Cabrera P. Tuberculosis. 2011; 26: 66-72.

29. Lasso BM. Meningitis tuberculosa: claves para su diagnóstico y propuestas terapéuticas. Rev Chil Infectol. 2011; 28: 238247.

30. American Thoracic Society, Centers for Disease Control and Prevention and Infectious Diseases Society of America. Treatment of Tuberculosis. MMWR. 2003; 52: 1-88. 